
males http://ow.ly/A0SjL

Sudhir Kurl ${ }^{1}$, Sae Young Jae ${ }^{2}$ and Jari A. Laukkanen ${ }^{1,3}$

${ }^{1}$ Institute of Public Health and Clinical Nutrition, School of Public Health and Clinical Nutrition, University of Eastern Finland, Kuopio, Finland. ${ }^{2}$ Dept of Sports Informatics, College of Arts and Physical Education, University of Seoul, South Korea. ${ }^{3}$ Dept of Internal Medicine, Lapland Central Hospital, Rovaniemi, Finland.

Correspondence: Sudhir Kurl, Institute of Public Health and Clinical Nutrition, Dept of Medicine, University of Eastern Finland, P.O. Box 1627, 70211 Kuopio, Kuopio, Finland. E-mail: Sudhir.kurl@uef.fi

Received: March 202014 | Accepted after revision: July 242014 | First published online: Aug 192014

Conflict of interest: None declared.

\title{
References
}

1 Friedman GD, Klatsky AL, Siegelaub AB. Lung function and risk of myocardial infarction and sudden cardiac death. N Engl J Med 1976; 294: 1071-1075.

2 Engström G, Lind P, Hedblad B, et al. Lung function and cardiovascular risk: relationship with inflammationsensitive plasma proteins. Circulation 2002; 106: 2555-2560.

3 Schünemann HJ, Dorn J, Grant BJ, et al. Pulmonary function is a long-term predictor of mortality in the general population: 29-year follow-up of the Buffalo Health Study. Chest 2000; 118: 656-664.

4 Bang KM, Gergen PJ, Kramer R, et al. The effect of pulmonary impairment on all-cause mortality in a national cohort. Chest 1993; 103: 536-540.

5 Sin DD, Wu L, Man SFP. The relationship between reduced lung function and cardiovascular mortality: a population-based study and a systematic review of the literature. Chest 2005; 127: 1952-1959.

6 Laukkanen JA, Mäkikallio TH, Rauramaa R, et al. Cardiorespiratory fitness is related to the risk of sudden cardiac death: a population-based follow-up study. J Am Coll Cardiol 2010; 56: 1476-1483.

7 Sparrow D, Weiss ST, Vokonas PS, et al. Forced vital capacity and the risk of hypertension. The Normative Aging Study. Am J Epidemiol 1988; 127: 734-741.

8 Pasceri V, Willerson JT, Yeh ET. Direct proinflammatory effect of C-reactive protein on human endothelial cells. Circulation 2000; 102: 2165-2168.

9 Zwaka TP, Hombach V, Torzewski J. C-reactive protein-mediated low density lipoprotein uptake by macrophages: implications for atherosclerosis. Circulation 2001; 103: 1194-1197.

10 Kannel WB, Wolf PA, Verter J. Manifestations of coronary disease predisposing to stroke. The Framingham study. JAMA 1983; 250: 2942-2946.

11 Cabanes LR, Weber SN, Matran R, et al. Bronchial hyperresponsiveness to methacholine in patients with impaired left ventricular function. N Engl J Med 1989; 320: 1317-1322.

12 Lange P, Groth S, Mortensen J, et al. Pulmonary function is influenced by heavy alcohol consumption. Am Rev Respir Dis 1988; 137: 1119-1123.

\section{Autonomic dysregulation: a mechanism of asthma death}

\author{
To the Editor:
}

Therapeutic advances in the management of asthma have led to a gradual but sustained reduction in mortality $[1,2]$. However, the death of otherwise healthy young individuals remains a tragic and all too frequent occurrence. The unheralded demise of two of our patients with previously minimally troublesome asthma led us to reconsider the mode of death in such patients. Numerous studies and retrospective reports have attributed such mortality to preventable factors such as inadequate severity assessment, discontinuity of medical care, poor concordance to prescribed therapies and poor management of acute asthma exacerbations [3,4]; others have attributed fatalities to significant behavioural, socioeconomic or psychosocial factors including smoking, denial, depression and alcohol abuse $[1,5,6]$.

Whilst these associations are undeniably important they do not adequately explain the observed clinical picture of precipitous deterioration, loss of consciousness, and death. RoBIN et al. [7] reported two patients with an abrupt demise, one during a telephone conversation and with no prior asthma-related symptoms. Grubb et al. [8] followed a teenage boy with a history of long-standing asthma after repeated near fatal asthma episodes requiring resuscitation. Our similar experience of two unexpected fatalities caused us to 
question bronchoconstriction as the primary mode of death. Others have suggested that vasovagallyinduced bradycardia results in a potentially lethal syncope in predisposed individuals. We have sought to test this hypothesis in subjects from our asthma clinic who have a history of unconsciousness precipitated by exacerbations using the simulated diving reflex of facial immersion to replicate such events [9].

Participants were recruited through the Academic and Outpatient Respiratory department at Castle Hill Hospital (Cottingham, UK) over a 12-month period. Subjects were excluded if they had significant cardiovascular or neurological comorbidities, a pacemaker, were taking beta-blockers or calcium channel antagonists, or a history of upper airway infection in the past 4 weeks. All participants gave written informed consent and the study was approved by the Hull and East Riding Local Research Ethics committee (LREC 12/YH/0520).

There were three arms to the study: 1) 10 subjects without a history of prior medical problems (control group); 2) 10 subjects with a diagnosis of asthma on treatment step 3 as defined by the British Thoracic Society (BTS) guidelines (asthmatic group) [10]; and 3) five subjects with a prior history of unexplained syncope during an asthma exacerbation on treatment step 3 of the BTS guidelines (syncopal asthmatics). Each participant underwent the same dive reflex protocol outlined below.

Participants were monitored using a Nexfin (Model 1, Bmeye; Amsterdam, the Netherlands) recorder and a 12-lead ECG for a period of $2 \mathrm{~min}$ at rest. They were asked to perform a "dive" lasting $30 \mathrm{~s}$. From a sitting position the face was immersed in a bowl of $12^{\circ} \mathrm{C}$ water, with one preparatory breath and no preceding hyperventilation. In the event that a participant was unable to complete a 30 -s dive after three attempts, their longest effort was used for analysis. Recording was continued for 2 min post dive.

The Nexfin derives a beat-to-beat estimation of heart rate, and both systolic and diastolic blood pressures from a finger sensor. These data were transferred in their entirety onto spreadsheets and analysed in $10 \mathrm{~s}$ epochs. Mean values for heart rate, systolic (SBP), diastolic (DBP) and mean arterial (MAP) blood pressures were computed. The 12-lead ECGs were manually measured using CardioSoft (GE Healthcare, Hatfield, UK) software, giving beat-to-beat values for RR and QT. A second value for heart rate was calculated from $\mathrm{RR}$, and QT was corrected using Bazett's formula to calculate QT interval corrected for heart rate (QTc). The pulse rate data recorded by the Nexfin were used in the statistical analysis and validated against the CardioSoft heart rate recordings.

From the study by HiEBERT et al. [9] we calculated the study sample size to be 25-30 with ten control and asthmatic participants, and 5-10 asthmatics with a history of unexplained collapse. One-way ANOVA would have $80 \%$ power to detect at the 0.05 level an effect size of 0.36 (nQuery Advisor, Statistical Solutions Ltd, Cork, Ireland). It was estimated that there would be two dropouts in each group. Change in heart rate induced by the dive was the primary outcome for our study. Data is reported as the mean $\pm \mathrm{SD}$ difference from pre-dive baseline in all parameters assessed. Area under the curve (AUC) analysis of the dive was conducted using the trapezoidal rule. Comparison at peak response $(+10 \mathrm{~s})$ was by two-tailed t-test and ANOVA (one-way) performed for the overall cohort during the dive. Significance was assumed at the 5\% level and all analyses were performed with the SPSS for windows version 18.0 (SPSS Inc., Chicago, IL, USA).

25 subjects fulfilled the inclusion criteria and simulated dive protocol; 10 controls, 10 asthmatics and five syncopal asthmatics. It should be noted that, despite scrutiny of the inclusion/exclusion criteria, one of the subjects recruited into the syncopal asthmatic group was subsequently found to have Holmes-Adie syndrome and was excluded from the analyses.

There were consistent and reproducible responses to the dive protocol in all three groups. Significant differences in change in heart rate between the syncopal asthmatic and control groups were noted in both the AUC over the 30 -s period of water immersion $(\mathrm{p}=0.04)$ and the peak tachycardia and immediate bradycardia induced by immersion $(\mathrm{p}=0.03)$. No significant difference was observed between the responses of the two asthmatic groups (fig. 1a). Similar statistical differences were noted in the QTc; however, no subject exhibited a pathological prolongation (fig. 1b).

All groups showed the typical change in blood pressure in response to a dive and there were no significant differences noted between any of the groups. There were no adverse events reported in the study.

The use of high-dose or potent $\beta_{2}$-agonists, inappropriate patient management, and significant behavioural, socioeconomic or psychosocial factors have been implicated in unexpected asthma deaths by various groups $[1,4-6]$. To invoke "lifestyle" as an explanation does not aid in our understanding of the pathophysiology of these events. Necroptic studies and case series' have identified that sudden demise of asthmatics is not always related to the severity of asthma or to having the typical pathophysiological changes seen in prolonged status asthmaticus $[7,8,11,12]$. This raises doubt over the suggestion that severe bronchospasm 

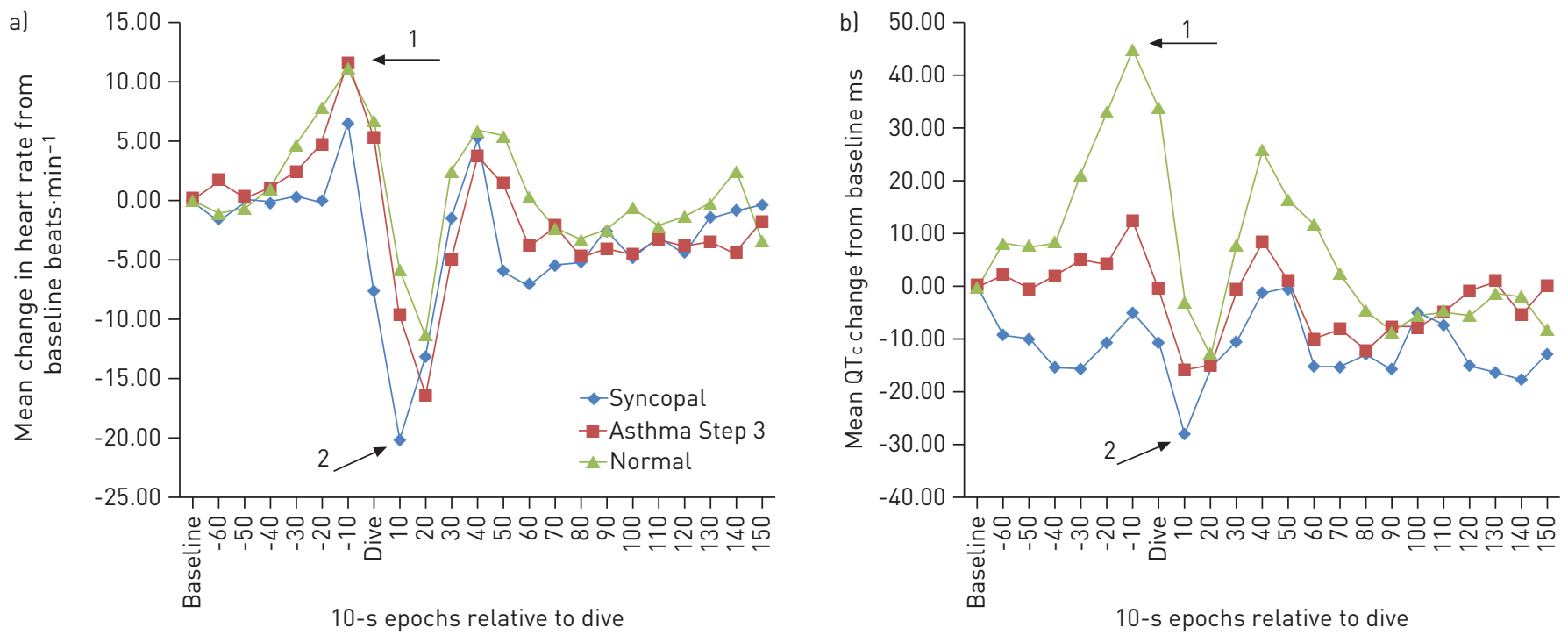

FIGURE 1 a) Mean change in heart rate from baseline throughout the dive protocol. Each point represents mean group value over a 10-s period. The arrows highlight time-points where 1 ) there is a variation in pre-immersion tachycardia (dive $-10 \mathrm{~s}$ ) and 2) immediate post-immersion bradycardia (dive $+10 \mathrm{~s}$ ) between the subjects. b) Mean change in QT interval corrected for heart rate (QTc) from baseline throughout the dive protocol. Each point represents mean group value

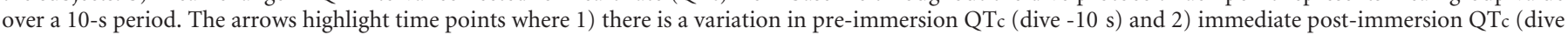
$+10 \mathrm{~s}$ ) between the syncopal asthma subjects and normal subjects.

is the sole mechanism of these fatalities. It is possible that sudden death in asthma may be a manifestation of cardiovascular rather than respiratory pathophysiology.

In our study we have attempted to test the hypothesis that bradycardia may be a relevant autonomic dysregulatory mechanism to describe collapse or fatalities some patients with asthma. Clearly, such a hypothesis is impossible to test in the post mortem situation and we have used as a surrogate patients under our care who have a history of syncope during acute episodes. To our knowledge this subset of patients has not previously been studied, possibly because unconsciousness may simply be assumed to be due to asphyxia. Alternatively cough syncope may be invoked. Cough syncope has been described for over a century. The traditional mechanism, loss of consciousness due to an increase in intrathoracic and/or cerebrospinal fluid pressure, is now regarded as inadequate [13]. A neural-mediated reflex, distinct from carotid sinus syndrome or vasovagal syncope, resulting in a vasodepressor-bradycardia and ensuing syncope has been implicated [14]. The findings of our study suggest that a similar syndrome may occur in asthma.

The major observation of our study, that asthmatics with a preceding history of syncope, have a blunted tachycardia on initiation of a dive and that this relative bradycardia continues throughout the 30-s stimulation of the diving reflex suggests autonomic dysregulation either through increased vagal tone or a failure of sympathetic drive. This was a preliminary examination and not designed to definitively test the mechanisms underlying any observed change. More invasive and detailed studies will be required to determine pathophysiological mechanisms. Interestingly, we did observe that there was no change QTc prolongation, which is thought to be protective of arrhythmias during bradycardia. However, other diving reflex assessments have reported no alteration in the QTc on cold water facial immersions [15]. Thus, although the autonomic system has a clear influence on QT interval, it remains unclear as to whether the sympathetic or parasympathetic autonomic activity induced by the diving reflex exerts its own effects on QT interval in addition to heart rate [16].

Tests of autonomic function are notoriously difficult to evaluate. Although a number of methods exist to assess autonomic function [17], in our study we used the validated, reliable and practical test of the dynamic cardiovascular response to an abrupt stimulus, the diving reflex. Its limitations include: 1) the involvement of multiple receptors and afferent pathways during these manoeuvres; and 2) that repeated manoeuvres may result in habituation or short-term training and hence may result in a more profound bradycardia [18, 19].

There are a number of limitations in our study. Recruitment was slower than expected in both asthmatic groups because of a perception that facial immersion could lead to worsening of asthma symptoms. All the subjects were aware of the study hypothesis and we cannot exclude some functional or autonomic influence on the results seen. That the observations in both the control asthmatics and healthy volunteers had similar trends in the parameters assessed makes this less likely. The major criticism of this study is the small number 
of subjects and while we have shown statistical (and we believe clinically) significant effects studies with larger numbers of subjects would be needed to confirm and extend these observations.

In this study we report the first experimental data showing that in patients with a history of asthma and collapse there may be an inherent autonomic dysregulation. Although our numbers of subjects were small and larger evaluations are needed, it may be postulated that neutrally-induced cardiovascular dysfunction may result in some of these tragic and precipitate events.

0 @ERSpublications

Initial data show in patients with a history of asthma and collapse there may be an inherent autonomic dysregulation http://ow.ly/zj3JW

Jaymin B. Morjaria, Tim Rowland, Puneet Tailor, Shaan Hyder, Caroline E. Wright, Simon P. Hart and Alyn H. Morice Dept of Academic Respiratory Medicine, Hull York Medical School, University of Hull, Castle Hill Hospital, Cottingham, UK.

Correspondence: Jaymin B. Morjaria, Dept of Academic Respiratory Medicine, Castle Hill Hospital, Castle Road, Cottingham, East Yorkshire, HU16 5JQ, UK. E-mail: jaymin.morjaria@hey.nhs.uk

Received: April 122014 | Accepted after revision: June 12 2014 | First published online: Aug 192014

Clinical trial: This study is registered at ClinicalTrials.gov with identifier number NCT02083029.

Conflict of interest: None declared.

Acknowledgements: We acknowledge the Academic Cardiology Department of the University of Hull for allowing us to use the Nexfin machines.

\section{References}

1 Bergström SE, Boman G, Eriksson L, et al. Asthma mortality among Swedish children and young adults, a 10-year study. Respir Med 2008; 102: 1335-1341.

2 Global Initiative for Asthma. Global Strategy for Asthma Management and Prevention. GINA, 2014. www. ginasthma.org/documents/4 Date last updated: May, 2014 Date last accessed: October 15, 2013.

3 Rea HH, Garrett JE, Mulder J, et al. Emergency room care of asthmatics: a comparison between Auckland and Toronto. Ann Allergy 1991; 66: 48-52.

4 Spitzer WO, Suissa S, Ernst P, et al. The use of beta-agonists and the risk of death and near death from asthma. N Engl J Med 1992; 326: 501-506.

5 Harrison B, Stephenson P, Mohan G, et al. An ongoing confidential enquiry into asthma deaths in the Eastern Region of the UK, 2001-2003. Prim Care Respir J 2005; 14: 303-313.

6 Caponnetto P, Auditore R, Russo C, et al. "Dangerous relationships": asthma and substance abuse. J Addict Dis 2013; 32: 158-167.

7 Robin ED, Lewiston N. Unexpected, unexplained sudden death in young asthmatic subjects. Chest 1989; 96: 790-793.

8 Grubb BP, Wolfe DA, Nelson LA, et al. Malignant vasovagally mediated hypotension and bradycardia: a possible cause of sudden death in young patients with asthma. Pediatrics 1992; 90: 983-986.

9 Hiebert SM, Burch E. Simulated human diving and heart rate: making the most of the diving response as a laboratory exercise. Adv Physiol Educ 2003; 27: 130-45.

10 British Thoracic Society, Scottish Intercollegiate Guidelines Network. 101 British Guidelines on the Management of Asthma: a National Clinical Guideline. May, 2008 revised January, 2012. London, BTS, 2012.

11 Carroll N, Carello S, Cooke C, et al. Airway structure and inflammatory cells in fatal attacks of asthma. Eur Respir J 1996; 9: 709-715.

12 Saetta M, Di Stefano A, Rosina C, et al. Quantitative structural analysis of peripheral airways and arteries in sudden fatal asthma. Am Rev Respir Dis 1991; 143: 138-143.

13 Dicpinigaitis PV, Lim L, Farmakidis C. Cough syncope. Respir Med 2014; 108: 244-251.

14 Benditt DG, Samniah N, Pham S, et al. Effect of cough on heart rate and blood pressure in patients with "cough syncope". Heart Rhythm 2005; 2: 807-813.

15 Olsen CR, Fanestil DD, Scholander PF. Some effects of breath holding and apneic underwater diving on cardiac rhythm in man. J Appl Physiol 1962; 17: 461-466.

16 Ahnve S, Vallin H. Influence of heart rate and inhibition of autonomic tone on the QT interval. Circulation 1982; 65: 435-439.

17 Arnold RW, Dyer JA, Gould AB Jr, et al. Sensitivity to vasovagal maneuvers in normal children and adults. Mayo Clin Proc 1991; 66: 797-804.

18 Gallo L Jr, Maciel BC, Manco JC, et al. Limitations of facial immersion as a test of parasympathetic activity in man. J Physiol 1988; 396: 1-10.

19 Schagatay E, van Kampen M, Emanuelsson S, et al. Effects of physical and apnea training on apneic time and the diving response in humans. Eur J Appl Physiol 2000; 82: 161-169. 